\title{
Trajectoires familiales, scolaires et amoureuses : comment les mineurs incarcérés traversent leur jeunesse
}

Family, school and love trajectories: how incarcerated minors go through their youth

Yaëlle Amsellem-Mainguy, Benoît Coquard et Arthur Vuattoux

\section{(2) OpenEdition}

\section{Édition électronique}

URL : https://journals.openedition.org/popvuln/799

DOI : 10.4000/popvuln.799

ISSN : 2650-7684

Éditeur

LIR3S - Laboratoire Interdisciplinaire de Recherche "Sociétés Sensibilités Soin" (UMR 7366 CNRS-uB)

\section{Édition imprimée}

Date de publication : 1 juin 2018

Pagination : 45-73

ISBN : 978-2-918173-23-6

ISSN : 2269-0182

Référence électronique

Yaëlle Amsellem-Mainguy, Benoît Coquard et Arthur Vuattoux, "Trajectoires familiales, scolaires et amoureuses : comment les mineurs incarcérés traversent leur jeunesse », Populations vulnérables [En ligne], 4 | 2018, mis en ligne le 12 juillet 2019, consulté le 26 février 2022. URL : http:// journals.openedition.org/popvuln/799; DOI : https://doi.org/10.4000/popvuln.799

Ce document a été généré automatiquement le 26 février 2022.

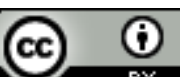

Les contenus de la revue Populations vulnérables sont mis à disposition selon les termes de la Licence Creative Commons Attribution 4.0 International 


\section{Trajectoires familiales, scolaires et amoureuses : comment les mineurs incarcérés traversent leur jeunesse}

Family, school and love trajectories: how incarcerated minors go through their youth

Yaëlle Amsellem-Mainguy, Benoît Coquard et Arthur Vuattoux

\section{Introduction}

1 Les mineurs détenus renvoient à l'image même de la jeunesse "à problèmes " et «dangereuse » au sein des classes populaires (Mauger, 2006 ; Beaud et Pialoux, 2003). Une fille ou un garçon en prison seraient de celles et ceux dont on ne veut plus dans les autres institutions de contrôle de la jeunesse, et ayant commis des actes qui ne relèveraient que de leur seule responsabilité (Fréchon et Robette, 2013; Vuattoux, 2014 ; Teillet, 2016). Cette représentation trouve d'ailleurs l'une de ses sources dans le droit, puisque l'ordonnance du 2 février 1945, qui régit aujourd'hui encore la justice pénale des mineurs, présente la prison comme un dernier recours, après que toutes les autres solutions ont été épuisées : la prison est ainsi la figure repoussoir d'une justice des mineurs d'ordinaire protectrice, en théorie compréhensive des situations sociales des mineurs. En conséquence, les détenus mineurs sont souvent décrits, dans des discours courants (parfois repris par des approches scientifiques de la criminalité), sous les traits d'individus « perturbés », autant « incasables » qu'« irrécupérables ».

2 Au-delà de ces représentations, une approche sociologique des parcours de ces jeunes nous enseigne comment ils peuvent être assignés à leurs casiers judiciaires et stigmatisés en retour. Nous avons fait le choix de mener une enquête qualitative qui porte prioritairement sur le rapport à la sexualité des jeunes incarcérés, donc des dimensions non directement judiciaires de leurs trajectoires (voir à ce sujet Chantraine, 2008). Ce décentrement du carcéral nous a amenés à les comparer aux autres jeunes de leur génération, tout en nous intéressant aux spécificités liées à leur expérience de 
prise en charge et de détention. Penser leur inscription dans une génération, en lien avec les travaux existants en sociologie de la jeunesse, peut permettre d'aller au-delà des visions souvent fantasmées du public carcéral dès lors qu'il est traité comme une somme d'individualités exceptionnelles, sous l'angle d'une sous-culture carcérale qu'il conviendrait d'étudier "à part ", en minorant les continuités entre le dedans et le dehors d'institutions parfois qualifiées de "totales» (Goffman, 1968). Se décentrer du carcéral permet en définitive de considérer les jeunes détenus comme des individus dans une situation exceptionnelle de détention, et non ancrés d'emblée dans une prétendue culture carcérale juvénile (Le Caisne, 2008). En effet, les trajectoires sociales et rapports au monde des jeunes détenus peuvent renvoyer à des profils qui ont beaucoup en commun avec ceux des jeunes de classes populaires de leur génération.

Dans cet article, nous voulons montrer ce que ces jeunes ont de «normal » selon leur âge et leurs origines sociales, au-delà des représentations qui font d'eux des "déviants ». Puis il s'agira de questionner certaines de leurs spécificités par le biais des situations récurrentes de vulnérabilité. Nous tenterons ainsi de faire la part des choses entre les vulnérabilités qui relèvent de l'incarcération (coupure de liens affectifs notamment) et celles qui relèvent du parcours antérieur à la détention (Goyette et Fréchon 2013). Il s'agit donc de comprendre l'expérience carcérale de ces jeunes selon leurs trajectoires. Quel lien peut-on faire entre les trajectoires des mineurs incarcérés et leurs plus ou moins grandes vulnérabilités face à l'expérience de l'incarcération ? Et dans quelle mesure l'expérience de la prison (répétée ou non) coïncide-t-elle avec certaines ruptures ou bifurcations biographiques (Bessin, Bidart et Grossetti, 2010) ?

4 De cette manière, l'article traite des conséquences des ruptures biographiques, scolaires et amoureuses induites par la ou les incarcérations successives. Nous verrons que sur ces thématiques il existe de grandes différences au sein d'un groupe que l'on aurait tendance à homogénéiser. Nous insisterons notamment sur les logiques de différenciation liées aux parcours scolaires, entre ceux qui ont arrêté l'école en primaire ou début de collège, et ceux qui sont allés jusqu'au lycée en filière générale (Palheta, 2012). Cet aspect scolaire des trajectoires sociales a d'ailleurs permis, durant les entretiens, de mettre à l'épreuve la réflexivité et le sens de la place de ces jeunes vis-à-vis des autres de leur génération. Les « décrocheurs " par exemple (Millet et Thin, 2005), qui ont très tôt été orientés vers des voies de relégation ou désignés comme des " délinquants » ou " jeunes à problème ", sont les plus prompts à se situer comme " en dehors » de la jeunesse en disant par exemple : « la jeunesse, c'est les autres». Tandis que ceux qui fréquentaient encore l'école au moment de leur incarcération partagent davantage les codes de leur génération et voient plus la prison comme un stade transitoire, qui ne marquera que leur jeunesse, mais pas forcément leur "vie d'adulte ». L'autre thématique transversale retenue dans les extraits d'entretiens mobilisés est celle de l'entrée dans la sexualité et les relations conjugales (Maillochon, 2001). Sur ce point, les jeunes filles et garçons rencontrés sont assez semblables à celles et ceux de leur milieu social (Clair, 2008 ; Amsellem-Mainguy et Dumollard, 2015), surtout si l'on compare leurs propos aux enquêtes dans les sections d'enseignement spécialisé (SEGPA) où sont surreprésentés les jeunes de classes populaires (Berger et al., 2015). Par contre, en ce domaine, l'incarcération amène une spécificité. Celle-ci apparaît comme une épreuve dans la gestion de leurs relations amoureuses, amicales et familiales. L'emprisonnement, selon la " gravité » des peines (motifs de condamnation et durée de la peine), tend à pousser ces jeunes à recomposer leurs attentes en matière de relations 
amoureuses (mais aussi amicales) et favorise un rapport aux autres marqué par la suspicion et l'auto-exclusion.

\section{Encadré 1. Présentation de l'enquête}

L'enquête s'est déroulée de novembre 2015 à avril 2017, comprenant une trentaine de jours sur le terrain dans cinq prisons françaises ${ }^{1}$. Durant cette période, nous avons effectué des entretiens individuels avec 72 jeunes détenus (62 garçons et 10 filles), âgés de 14 à 18 ans, ainsi que 59 professionnels issus des quatre principales administrations présentes en détention auprès des mineurs : 26 éducateurs, 19 surveillants, 8 enseignants et 6 professionnels de santé 2 . Enfin, nous avons effectué des focus groupes lors de la restitution des résultats auprès de 51 jeunes et de 66 professionnels. La restitution, organisée avant la remise du rapport de l'enquête, permettait notamment de tester des hypothèses, de revenir sur certains sujets peu abordés lors des entretiens individuels et plus généralement de discuter les premières orientations de la recherche auprès des personnes concernées au premier chef par celle-ci.

Les cinq prisons enquêtées sont réparties sur l'ensemble du territoire national métropolitain, afin d'accéder à une compréhension de certaines réalités locales, d'effets de contexte. Deux prisons sont situées en Île-de-France, les trois autres dans d'autres régions ${ }^{3}$. Parmi ces cinq prisons, trois sont des Maisons d'arrêt possédant des « quartiers mineurs » $(\mathrm{QM})$, c'est-à-dire des quartiers réservés aux mineurs au sein de prisons accueillant également des majeurs, l'une des deux maisons d'arrêt possédant un quartier mineures pour femmes. Deux autres prisons sont des Établissements pénitentiaires pour mineurs (EPM), c'est-à-dire des structures carcérales accueillant exclusivement des mineurs et bénéficiant d'un renfort en éducateurs (la gestion quotidienne étant effectuée par des " binômes » éducateurs/surveillants présents ensemble, mais seulement en journée, dans les unités) $)^{4}$.

Si l'objet principal de cette recherche était de comprendre le rapport à la vie affective et sexuelle de ces jeunes, mais également la gestion de la santé et de la sexualité des jeunes par les personnels qui les encadrent, nous avons exploré différentes dimensions des vies des jeunes détenus, dont leur rapport à l'amour, à la conjugalité, au travail et à la mobilité.

\section{Des prisons de classes populaires}

Les sciences sociales ont montré comment les discours autour d'une prétendue augmentation de la délinquance des mineurs ont servi à déployer un dispositif pénal en direction des jeunes de classes populaires (Sallée, 2016). L'inflexion législative récente est notamment marquée par la loi Perben I en 2002 qui entérine la création des premiers établissements pénitentiaires pour mineurs (EPM). Six de ces prisons pour mineurs âgés de 13 à 18 ans ont ainsi été ouvertes en France entre 2007 et 2008. Ces EPM s'ajoutent ainsi aux « quartiers mineurs ", qui sont des ailes réservées aux mineurs dans des prisons pour majeurs. Au $1^{\mathrm{er}}$ mai 2017, on dénombre 69679 personnes incarcérées (dont 2379 femmes), dont 792 mineurs âgés (dont 46 filles) de 13 à 18 ans 
(avec une surreprésentation notable des 16-17 ans). Les statistiques disponibles ${ }^{5}$ au $1^{\text {er }}$ janvier 2017 établissent un âge moyen des mineurs détenus de 16,43 ans (16,06 pour les filles contre 16,45 pour les garçons) et un âge médian des mineurs détenus de 17 ans (16 ans pour les filles contre 17 ans pour les garçons). Le nombre de mineurs incarcérés à un instant $t$ est resté relativement stable dans les dernières années, mais cette stabilité s'est accompagnée d'un plus fort recours aux alternatives répressives à la prison, à l'instar des placements en centres éducatifs fermés - eux aussi issus de la loi Perben. Les mineurs représentent ainsi près de $1,2 \%$ des détenus. Parmi eux, environ un tiers étaient incarcérés dans des établissements pénitentiaires pour mineurs (EPM) et les autres dans des maisons d'arrêt (pourvues le plus souvent de quartiers spécifiques pour les mineurs) ${ }^{6}$. Ces données ne permettent pas de connaitre précisément l'évolution de la part de filles mineures détenues, mais on sait qu'elles représentent environ $7 \%$ des peines de privation de liberté enregistrées au Casier judiciaire national ${ }^{7}$, et comme pour les majeures, une part encore moindre (de l'ordre de $3 \%$ ) des personnes effectivement incarcérées.

6 À partir des données que nous avons récoltées via les entretiens, un premier regard sur les origines sociales des détenus mineurs rappelle d'emblée que la prison est une institution qui s'emploie à "punir les pauvres» (Wacquant, 2004). À l'image de la population pénale (Combessie, 2009), les jeunes détenus, dans leur quasi-totalité, sont presque tous enfants d'employés, d'ouvriers, de chômeurs ou de travailleurs précaires à leur compte. Ceux que nous avons rencontrés en entretiens ne dérogent pas à la règle. De plus, ils vivent ou ont grandi pour une majorité d'entre eux dans des quartiers d'habitat social. Une autre caractéristique générale de cette population est qu'elle est surtout masculine (à l'image de la population détenue majeure). Enfin, dans la lignée de leur appartenance quasi systématique aux classes populaires, ils et elles sont souvent ${ }^{8}$ racisée'es en tant qu'immigrée's ou descendante's d'immigrés d'Afrique du Nord ou subsaharienne, et/ou associée's a un groupe stigmatisé (notamment roms, émigrés d'Europe de l'Est) ${ }^{9}$. L'idéal type du jeune incarcéré est donc celui d'un garçon de 17 ans, fils d'employés ou d'ouvriers immigrés, ayant grandi en ville dans les quartiers populaires. C'est ainsi une population qui, à première vue, pourrait paraître assez homogène, d'autant qu'elle occupe en quelque sorte la place de « minorité du pire » au sein d'un groupe ("jeunes de quartier», jeunes de classes populaires) lui-même stigmatisé.

Tableau 1. Tableau synthétique des enquêtés

\begin{tabular}{|c|c|c|}
\hline & Garçons $(n=60)$ & Filles $(n=12)$ \\
\hline Âge & $\begin{array}{l}\text { Les garçons ont en moyenne } 16,5 \text { ans. } 7 \\
\text { d'entre eux ont moins de } 16 \text { ans, } 53 \text { ont } \\
\text { plus de } 16 \text { ans. } \\
\text { Répartition par âge: } \\
14 \text { ans : } 2 \\
15 \text { ans : } 5 \\
16 \text { ans : } 17 \\
17 \text { ans : } 35 \\
18 \text { ans : } 1\end{array}$ & $\begin{array}{l}\text { Les filles ont en moyenne } 16,3 \text { ans. } 2 \\
\text { d'entre elles ont moins de } 16 \text { ans, } 10 \\
\text { ont plus de } 16 \text { ans. } \\
\text { Répartition par âge: } \\
14 \text { ans : } 1 \\
15 \text { ans : } 1 \\
16 \text { ans : } 3 \\
17 \text { ans : } 7 \\
18 \text { ans : } 0\end{array}$ \\
\hline
\end{tabular}




\begin{tabular}{|c|c|c|}
\hline $\begin{array}{lr}\text { Profession } & / \\
\text { Activité des } \\
\text { parents }\end{array}$ & \multicolumn{2}{|c|}{$\begin{array}{l}\text { Concernant l'ensemble des jeunes (filles et garçons), une majorité de leurs } \\
\text { parents ( } 39 \% \text { ) est sans activité professionnelle, notamment chômage et parents } \\
\text { (mères) au foyer. } 22 \% \text { des parents sont employés, } 18 \% \text { sont artisans ou à leur } \\
\text { compte, } 12 \% \text { sont ouvriers, } 7 \% \text { appartiennent aux professions intermédiaires. } \\
\text { Les } 2 \% \text { restant correspondent à des parents cadres ( } 1 \text { seul parent) ou retraités. } \\
\text { Ces chiffres ne prennent pas en compte les } 15 \% \text { de parents pour lesquels les jeunes ne } \\
\text { savaient pas nommer la profession ou le type d'activité. }\end{array}$} \\
\hline $\begin{array}{l}\text { Niveau } \\
\text { scolaire }\end{array}$ & $\begin{array}{l}7 \text { garçons ont un niveau correspondant à } \\
\text { l'enseignement primaire, } 31 \text { ont un } \\
\text { niveau collège, et } 16 \text { un niveau CAP ou } \\
\text { lycée (le plus souvent professionnel). Le } \\
\text { niveau scolaire n'a pas pu être renseigné } \\
\text { pour } 6 \text { des } 60 \text { jeunes. }\end{array}$ & $\begin{array}{l}2 \text { filles ont un niveau primaire, } 8 \text { un } \\
\text { niveau collège (essentiellement } 4^{\mathrm{e}} \text { et } \\
3^{\mathrm{e}} \text { ), et } 2 \text { un niveau lycée ou CAP. }\end{array}$ \\
\hline $\begin{array}{l}\text { Trajectoire } \\
\text { carcérale ou } \\
\text { de prise en } \\
\text { charge }\end{array}$ & $\begin{array}{l}19 \text { garçons ont déjà connu la détention } \\
\text { antérieurement à leur incarcération } \\
\text { actuelle, } 23 \text { ont été placés en } \\
\text { hébergement fermé/renforcé à la PJJ (CEF, } \\
\text { CER, etc.), } 28 \text { ont été placés en foyer (ASE } \\
\text { ou PJJ) et/ou suivis en milieu ouvert et } 16 \\
\text { n'ont connu aucune prise en charge } \\
\text { antérieur. Un même mineur peut cumuler } \\
\text { plusieurs de ces situations. }\end{array}$ & $\begin{array}{l}5 \text { filles ont déjà connu la détention } \\
\text { antérieurement à leur incarcération } \\
\text { actuelle, } 8 \text { ont été placées en } \\
\text { hébergement fermé/renforcé à la PJJ, } \\
2 \text { ont été placées en foyer et } 3 \text { n'ont } \\
\text { connu aucune prise en charge } \\
\text { antérieure. Une même mineure peut } \\
\text { cumuler plusieurs de ces situations. }\end{array}$ \\
\hline
\end{tabular}

7 Au-delà de leurs appartenances communes, ce tableau synthétique des enquêtés permet de cerner quelques inégalités familiales entre jeunes incarcérés. À partir de leurs déclarations, nous avons en effet constaté la diversité de leurs origines sociales. Néanmoins, ces chiffres ont été obtenus à partir des déclarations de la profession de leurs parents par les jeunes eux-mêmes. Ces déclarations sont parfois approximatives, et cette méconnaissance s'accentuait évidemment chez les jeunes qui n'avaient plus de contact avec leurs parents. Malgré tout, notre méthode de récolte de données déclaratives (nous remplissions une fiche synthétique durant l'entretien) nous donne un aperçu de la relative hétérogénéité des origines sociales parmi les jeunes incarcérés. Il en ressort que quatre jeunes enquêtés sur dix ont des parents sans activité professionnelle: ratio largement supérieur au taux de chômage sur le territoire national français, et supérieur aussi à la moyenne dans les quartiers dits « politique de la ville »(QPV). Mais il faut d'emblée être prudent sur sa pertinence. En effet, il arrivait qu'au cours de la conversation certains jeunes évoquent une autre activité parentale, tout en affichant une méconnaissance des conditions de travail, notamment des mères. Ces dernières, souvent désignées par les jeunes comme «mères au foyer » (et donc assimilées aux "sans activité professionnelle») peuvent en réalité travailler à temps partiel ou par intermittence, mais leurs enfants continuent pour autant de les voir «à la maison ». Autre biais déclaratif repéré, la part des parents déclarés "artisans ou à leur compte » $(18 \%)$ est étonnamment supérieure à la part des parents "ouvriers " (12\%), à l'inverse donc de la population générale. Il serait donc hasardeux d'interpréter ce chiffre outre mesure, compte tenu là encore des biais de déclaration. Lors des entretiens, nous avons en effet évoqué avec les jeunes détenus le travail formel, mais aussi informel de leurs parents. Typiquement, les pères qui exercent l'activité de 
«ferrailleur » de façon informelle auront été classés avec les « artisans ». Plus encore, on notera que $15 \%$ des jeunes ne connaissent pas la profession de leurs parents, même s'ils peuvent encore être en lien avec ces derniers. Certains verront peut-être dans cette approximation la preuve que les enfants ne sont pas proches de leurs parents. On pourrait aussi penser que les contrats précaires dans le salariat d'exécution amènent aujourd'hui à changer très régulièrement de travail, ou encore à avoir un métier peu valorisant auprès de ses proches, de telle sorte qu'on parle peu de son travail dans la sphère privée. Dans tous les cas, la perception subjective de la profession des parents montre, a minima, que l'ensemble de ces jeunes appartiennent aux classes populaires et que, dans le même temps, ils se situent objectivement et subjectivement dans les fractions les précaires (même si pas forcément les plus démunies) de ces classes. Nous observons par ailleurs qu'au sein de cette population, il existe de fortes inégalités de ressources (économiques, culturelles, scolaires, sociales) qui se retrouvent dans des configurations familiales plus ou moins précaires. Une partie des détenus n'ont que très peu (voire plus du tout) de contact avec leurs parents (souvent les parents séparés et les plus pauvres du panel), quand d'autres vivent encore dans le foyer familial avant incarcération, avec deux parents actifs et un couple stable. Non seulement ces différences expliquent des trajectoires variées, mais plus encore, elles impliquent des expériences carcérales différentes, notamment en termes de vulnérabilités liées à l'incarcération.

8 Le tableau précédent montre aussi que la part des jeunes ayant déjà connu la détention est bel et bien minoritaire (19/60 chez les garçons, 5/12 chez les filles). C'est là un chiffre important, d'autant que nous sommes face à des mineurs âgés en moyenne de 16,5 ans (42 enquêtée's sur 72 avaient 17 ans). En ce sens, l'idée que le public carcéral serait caractérisé par des longs suivis ASE ou PJJ et que la prison serait un dernier recours est à nuancer, du moins chez les garçons (28/60 des garçons, 10/12 des filles ont déclaré avoir déjà été pris en charge ASE et/ou PJJ antérieurement à la prison). Près de la moitié des garçons et deux filles étaient donc en prison pour, comme ils disent, leur "première affaire", en écho au contexte de pénalisation croissante d'actes délictueux qui ne donnaient pas forcément lieu à des peines de prison ferme voici quelques années (Bonelli, 2003).

9 Or, les entretiens révèlent une adaptation différente à l'incarcération de ceux qui ne figuraient pas dans la population prise en charge au préalable par la PJJ. Cette adaptation dépend bien sûr des fonctionnements des établissements, mais en ce qui concerne les variations propres aux détenus elle va dépendre des faits retenus pour l'incarcération, de l'interconnaissance dans la détention (Bony, 2015) et d'autres paramètres individuels liés aux trajectoires biographiques. En règle générale, elle sera potentiellement plus brutale pour ceux envoyés directement en prison que pour les jeunes ayant connu le milieu ouvert PJJ. Dans ce cadre en effet, l'incarcération peut constituer une menace de sanction en dernier recours et de fait être présente dans la tête des jeunes (pour exemple les fugues de Centres éducatifs fermés - CEF - impliquent potentiellement une sanction par l'incarcération). De plus, fréquenter des établissements de prise en charge judiciaire augmente les chances de connaître d'autres jeunes ayant déjà été incarcérés et qui racontent la prison, la décrivent dans son architecture comme dans son fonctionnement, et autant de discussions collectives qui contribuent aux capacités individuelles à s'y adapter (Le Caisne, 2008). En cela, la vulnérabilité des jeunes incarcérés rencontrés s'accentue plus ou moins pendant et 
après la détention, en fonction des ressources sociales, amicales, familiales dont ils ont préalablement hérité et aussi de la configuration des rapports sociaux au sein de la prison, qui permet plus ou moins des situations d'entraides.

10 Enfin, le tableau présentant la population de jeunes enquêtés montre que la grande majorité des détenus rencontrés ont un niveau collège. De plus, il apparaît dans les entretiens que celles et ceux qui avaient « décroché » durant la période précédant leur incarcération ont tout de même été en contact avec l'école. Ils fréquentaient leurs anciens camarades de classe, ou passaient du temps près des établissements scolaires, comme l'avait déjà observé la sociologie de "l'école buissonnière » (Douat, 2011). Sans surprise, les enquêtés qui étaient lycéens, parfois en filière générale, appartenaient aussi à des familles plus stables que ceux qui étaient déscolarisés depuis les premières années du collège et avaient connu un long suivi ASE. On notera alors, contre une vision assez répandue, que ces jeunes n'appartiennent pas majoritairement à des familles monoparentales et ont beaucoup fait référence en entretien à l'autorité que leurs parents pouvaient encore exercer sur eux. En cela, les jeunes étaient assez similaires à ceux interrogés en milieu scolaire (collège ou lycée). Les parents représentent une autorité distante, et sont par ailleurs considérés comme les référents et supports les plus solides en divers domaines. Pour exemple, sur les questions relatives à la sexualité et la vie amoureuse, les enquêtés avaient tendance, comme la plupart des jeunes, à dire qu'ils préfèrent en parler avec leurs pairs, tout en considérant que la meilleure source d'information demeure leur famille, et plus spécifiquement leurs parents. La référence aux parents, à l'environnement familial restreint, est d'ailleurs très présente, sur de nombreux sujets, dans les entretiens réalisés.

\section{Vivre l'incarcération comme une rupture}

11 Certes, les conditions de condamnation et les dispositions du détenu à vivre "plus ou moins difficilement " son incarcération renvoient à des facteurs microsociologiques qu'il conviendrait d'analyser dans le détail. Néanmoins, en complément de notre travail sur les parcours individuels à partir des entretiens longs, nous avons tenté de repérer des facteurs plus généraux quant au fait de vivre ou non son incarcération comme une rupture biographique, ou comme une redéfinition du sens de la place.

12 Une première variable importante repose sur l'interconnaissance éventuelle au sein même de la prison. Si les détenus impliqués dans les mêmes «affaires" sont généralement séparés géographiquement, il est fréquent que plusieurs jeunes du même quartier soient incarcérés dans le même établissement ${ }^{10}$. Le fait d'être connu et éventuellement d'être reconnu (d'avoir une «bonne réputation») avant même son arrivée en détention participe d'un "accueil favorable» ou du moins d'un interrogatoire moins ténu lors du passage aux arrivants, et ensuite une moindre mise à l'épreuve de la part des autres détenus. À l'inverse, les jeunes qui «ne connaissent personne » dans la détention auront davantage de difficultés et pourront être harcelés verbalement, psychologiquement et/ou physiquement. Le fait d'être plus ou moins bien «accueilli " au sein de la détention renvoie ainsi, en partie, aux relations avec les autres détenus, mais aussi, bien sûr, avec les professionnels. Les cas les plus problématiques étant ceux des garçons étiquetés comme agresseurs sexuels (les " pointeurs »). Ce soupçon pèse surtout sur ceux qui ont une durée de mandat de dépôt importante (situation typique pour ce genre de délits). Un enquêté raconte même 
comment un nouveau détenu suspecté de «s'inventer une vie » a été jusqu'à fournir une copie de son acte d'incarcération, signé du juge, pour prouver qu'il n'était pas « un pointeur ». Et dans le cas des jeunes qui sont effectivement inculpés pour agressions sexuelles, à la pression des autres détenus peut s'ajouter un traitement discriminatoire de la part des professionnels. Certains surveillants s'arrangeront pour que les autres détenus soient au courant de "l'affaire ", dans l'objectif (non-dit) qu'ils s'en prennent au détenu incriminé - par des insultes répétées depuis les cellules, ou des agressions lors des déplacements et des temps collectifs.

\section{Extrait d'entretien avec Noé}

En fait, l'ambiance elle est plus liée à ce que t'as fait et aux raisons pour lesquelles t'es là que le reste.

Ouais même pas en fait. Après si tu tombes là pour viol ouais, mais après...

En fait, il y a que le viol qui fait que c'est différent?

Ouais.

Et genre là, les gars, il y en a qui sont là pour viol ?

Il y en a un ouais.

Et ça tu le sais, tout le monde le sait?

Ouais, tout le monde le sait.

Et il se passe quoi du coup?

Il sort pas en promenade.

$14 \quad[. .$.

Tu l'as déjà croisé?

Ouais.

Par exemple où ça?

Pour aller au parloir.

Ah oui. Et il t'a dit quoi ?

Je l'ai giflé.

Pourquoi?

Parce que j'aime pas ces trucs moi. J'imagine que c'est ma sœur des fois. Ça pourrait être.

Et t'es sûr que c'est vrai?

Ouais.

Lui il a dit que c'était vrai ou... ?

Lui il dit que c'est pas vrai. Mais on a vu son dossier et tout ça.

Comment vous avez vu son dossier?

L'auxi $^{11}$.

Ah oui, l'auxi lui il a accès aux dossiers?

Il a pas accès, mais il peut aller voir en scred [en secret] comme ça vite fait et tout.

15 On peut aussi remarquer que les jeunes qui ne parviennent pas à s'adapter à la vie en détention, qui vont avoir par exemple des problèmes de sommeil repérés par l'unité sanitaire, vont être quasi systématiquement placés sous traitements médicamenteux. 
Or, cette banalisation des prescriptions entraîne, au sein de la détention, une somme d'enjeux autour des prises de médicaments, revenant à savoir «qui en prend? " et «pourquoi? ». Les médicaments sont justifiés par les détenus au motif que l'expérience carcérale est "dure », par contre, lorsque les consommateurs de "cachetons » sont déjà stigmatisés par les autres détenus, la prise du traitement rajoute à la violence qu'ils peuvent subir et participe aux lignes de partage entre détenus.

\section{Entretien avec Nathan}

Et tu fais des activités ? T'as pas école le matin?

Ils m'ont proposé de faire un test, j'ai fait un test, mais...

Et t'as envie d'y aller, à l'école?

Bah j'hésite en fait. Le matin je dors. J'arrive pas à dormir le soir. Je prends des médicaments pour dormir. Ils m'ont donné des médicaments, mais j'ai l'impression que ça fait rien...

Ça ne marche pas du tout?

Non...

Et tu sais pourquoi tu n'arrives pas à dormir ? Le bruit ?

Non, j'sais pas. J'suis allongé, j'sais pas quoi faire.

Et du coup t'as vu des médecins ici?

Oui, en bas, j'suis descendu, il y a une pharmacie, des médecins, je leur ai parlé, il m'ont donné des médicaments, mais on dirait c'est fait exprès pour faire croire que ça endort, mais ça m'endort pas du tout...

Tu penses que c'est de faux médicaments?

Ça donne même pas un tout petit peu envie de dormir, rien du tout...

Et avant d'être ici t'avais des problèmes de sommeil ?

Avant allongé je dormais d'un coup...

$[\ldots]$

La toute première fois que j'ai dormi ici, au début j'ai dormi d'un coup, alors qu'en garde à vue j'arrivais pas à dormir. Enfin j'arrivais à dormir, mais il y a des jours j'arrivais pas à dormir. J'ai fait 4 jours en garde à vue... Ici le premier jour que je suis venu ici j'ai dormi d'un coup après j'ai plus réussi à dormir...

Et tu sais pas trop pourquoi, c'est comme ça... C'est un autre cadre, des fois c'est difficile...

Moi j'arrive à m'habituer normalement. Là franchement je comprends pas...

C'est la première fois que tu viens en détention là?

Oui

Et avant t'as déjà eu des suivis, t'as déjà été placé ?

J'ai déjà été au commissariat, mais des suivis non...

18 A contrario, certains jeunes pris en charge par l'ASE depuis leur petite enfance pouvaient faire référence à la prison comme un lieu de vie auquel ils ont été habitués, même s'ils n'occupent pas forcément le haut de la hiérarchie symbolique entre détenus. Nous sommes pour certains profils dans un processus clair de socialisation par l'institution de la prison et autres établissements de prise en charge. L'un des jeunes interrogés nous expliquait même provoquer ses incarcérations successives dans un 
même EPM pour retrouver un cadre qui selon lui est « mieux » que son quartier et sa famille, reprenant ainsi à son compte les commentaires que lui font les professionnels à chacune de ses entrées. Les entretiens avec les jeunes pris en charge depuis longtemps et par des institutions multiples révélaient par ailleurs une forme d'adhésion aux explications que les encadrants leur inculquent à propos de leurs parcours de vie. Dans la même logique de récit institutionnel de soi, des détenus avaient tendance à "dérouler » leur casier judiciaire, quand d'autres étaient imprégnés des discours de leurs éducateurs et psychologues.

\section{Entretien avec Mounir}

Parce que du coup, toi, t'as été en CEF avant?

Moi j'ai fait tout ce qu'il y a de possible dans cette France en termes d'éducation.

\section{C'est-à-dire?}

J'ai fait foyer, famille d'accueil, EPE [établissement de placement éducatif], EPM [établissement pénitentiaire pour mineurs], prison, Paris en prison, transfert, établissement pénitentiaire pour mineur. J'ai fait plein de trucs. J'ai fait les EPE, les lieux de vie, tout ce...

Les CER [centre éducatif renforcé] et les CEF?

CER, CEF, au Maroc là-bas, partout. Séjour de rupture, j'ai été au Chili. J'ai tout fait.

Et alors, t'en tires quoi des expériences que t'as vécues?

Beh je serais pas là. Pour l'instant, on est à zéro.

Il y a rien qui a marché?

Beh non. Après, voilà, manque d'éducation quand t'es petit, tu grandis, t'as une prise de confiance c'est tout, c'est comme ça.

20 Là encore, les différences entre jeunes pris en charge auparavant ou non par l'ASE tiennent bien souvent au fait que certains, comme Mounir ci-dessus, ont des trajectoires très tôt heurtées par une rupture biographique importante (déménagement, famille éclatée, etc.), tandis que d'autres détenus ont connu une stabilité dans leur environnement, leurs parents, leurs groupes de pairs, leurs lieux de vie, et même leur scolarisation. En ce sens, le «choc » qui peut parfois caractériser l'expérience carcérale chez ceux qui y étaient a priori le moins destinés, semble compensé par d'autres ressources qui permettent de supporter la détention. Entre autres choses, les visites au parloir des parents seront plus fréquentes si ces derniers n'ont pas coupé les ponts avec leur enfant depuis longtemps. De même, ils pourront envoyer de l'argent pour cantiner des produits de base, ou encore fournir des vêtements lors des visites.

D'un autre côté, les jeunes ayant déjà eu un ou des membres de leur famille proche incarcérés auront plus de chance d'avoir un rapport « normalisé » à la prison, comme si là encore le passage par la détention faisait partie des possibles. Par contre, lorsque la prison se situe clairement dans une projection propre à la jeunesse, perçue comme âge de la vie transitoire vers un âge adulte plus stable et conforme. Cette distinction était très récurrente dans les entretiens et ne s'apparentait pas forcément aux discours institutionnels de réinsertion. Steven par exemple, 17 ans, insiste sur le caractère «tranquille » de l'incarcération en EPM en comparaison des conditions de détention beaucoup plus «dures » qu'aurait selon lui enduré son père lors d'une longue peine. Dans le même temps, il se projette vers une vie adulte différente de celle de son père, 
parce qu'il regrette d'être lui-même « un bébé parloir » et en conséquence ne veut pas «faire vivre » la même expérience à ses éventuels futurs enfants. Ici, la comparaison avec son père semble d'autant plus importante que Steven est dans une relation conjugale longue, avec perspective de mariage et d'enfants. Il n'exclut pas de " coffrer » (gagner de l'argent) illégalement dans le futur, mais par contre, son mode de vie familial et conjugal devrait selon lui être marqué par une stabilité et une respectabilité. En ce sens, l'expérience vécue de la prison, si elle est souvent abordée par la variable essentielle qu'est l'état (sanitaire, réglementaire) des lieux et l'action des professionnels, est aussi grandement dépendante des trajectoires antérieures des détenus ; ici, les mineurs semblent assez inégaux face aux conséquences qui peuvent en découler.

\section{Trajectoires scolaires et réflexivité}

D'autre part, les jeunes incarcérés, surtout ceux qui sont en EPM, ont l'école comme activité principale de leur emploi du temps. Certains ne seront jamais « autant allés à l'école » que lorsqu'ils passent par la prison, selon l'un des arguments de justification des éducateurs et plus encore des enseignants qui travaillent en EPM. La prison pourrait ainsi pallier des carences éducatives ayant mené aux situations que connaissent les jeunes. En EPM comme en QM, les cours priment sur les autres activités, y compris les entretiens avec la PJJ - à l'exception cependant de la santé (rendez-vous médicaux, extractions, etc.). On peut être privé de télé, ou sanctionné de différentes manières (jusqu'au placement au "mitard »), mais en aucun cas un détenu ne peut être privé de cours. Ainsi lors des entretiens, l'école revient tout autant dans le récit de la trajectoire antérieure que dans celui de la vie présente en prison.

\section{Entretien avec Adrien}

Et comment tu passes ta journée ici ? Qu'est-ce que tu fous ?

Tu veux dire ma journée entière?

Ouais, décris-moi une journée typique d'un mec en taule comme toi.

Moi ce que je fais... Déjà, je me réveille, je prends ma douche et tout. Mais tu vois, ils tapent dans ta porte à $7 \mathrm{~h} 30$, tu te réveilles un peu, tu prends ta douche en vitesse, je fais vite fait 50 pompes avant de descendre.

\section{0 pompes par jour tu fais?}

Non pas par jour, juste pour descendre, je fais vite fait des séries de 20. Je descends, je mange. Après tu vois, il y a l'école. Si tu veux y aller t'y vas, si tu veux pas y aller tant pis.

Des fois t'y vas pas non?

Si j'y ai toujours été moi.

Même quand ça te saoule?

Ouais, parce qu'après ils enlèvent les télés.

Ouais, c'est la carotte quoi.

Après, dès que tu rentres, c'est à $11 \mathrm{~h}$, c'est soit la promenade, ou soit t'attends, t'attends $13 \mathrm{~h}, 13 \mathrm{~h}$ tu manges, $14 \mathrm{~h}$, il y a re-l'école pendant deux heures. À $16 \mathrm{~h}$ c'est 
soit t'as ta promenade soit t'as rien. Et à $18 \mathrm{~h} 30$ tu manges. Et après ta journée elle est finie.

À 18 h30 du coup tu fais quoi ? Télé?

Moi j'aime bien c'est regarder les Ch'tis ${ }^{12}$.

En prison, l'école est avant tout vue comme une activité qui permet de sortir de sa cellule. Les groupes scolaires sont moins constitués en fonction des niveaux qu'en fonction des "compatibilités » entre détenus, pour éviter le chahut, les bagarres ou simplement les échanges (entre ceux qui n'ont pas le droit de se croiser sur ordonnance du juge). Dans cette logique, l'école en prison, bien qu'elle soit très importante dans le déroulement des journées (son absence lors des vacances scolaires se ressent tout particulièrement), n'apparaît pas comme une institution socialisatrice centrale à l'inverse de l'école « dehors ». D'ailleurs, les détenus ne parlent pas de «l'école », mais simplement des « cours » ou de « la classe ». Il s'agit concrètement, en allant en cours, de se rendre au lieu d'activité de la détention, toujours perçu comme un lieu de privation de liberté avant d'être un espace de sociabilité entre jeunes qui serait ainsi comparable à l'école « dehors ». Un autre aspect important dans le fait que les cours en prison ne sont pas "l'école", c'est la non-mixité (dans la grande majorité des établissements). Fréquemment, en entretiens et lors des focus groupes, les jeunes notaient que cette non-mixité rappelait qu'ils étaient ici dans un lieu de relégation et considérés comme des jeunes si peu recommandables qu'on ne peut les autoriser à vivre en mixité.

Surtout, de manière plus générale, l'école «dehors » est vue par eux comme un lieu marquant en ce qu'elle est aussi et surtout un lieu de classements en tous genres. Pour les détenus, la scolarisation reste ainsi la différence la plus évoquée entre eux et « les autres » de leur génération.

En effet, comme nous avons pu le voir en entretiens et aussi lors des restitutions collectives, « la jeunesse » ou « les jeunes », ou encore « les filles bien » sont décrits par les mineurs incarcérés sous les traits des jeunes de leur âge encore scolarisés dans les filières selon eux "normales", autrement dit, les collèges et lycées de filières générales, voire les étudiants. Et puisque les jeunes détenus sont principalement issus de quartiers populaires en périphérie, la jeunesse «bien» est aussi celle du « centreville ", lieu investi par eux sur un mode récréatif, en comparaison du quartier du quotidien (Lepoutre, 1997). Ces modèles de jeunes «bien », "qui sortent en ville ", " marchent avec leur sac à dos", etc., sont ainsi mobilisés par les détenus dans des moments de réflexivité où ils se situent au sein du monde social et plus précisément de leur génération. Il semble en ce sens que l'école participe, même dans leur cas, d'un véritable « tri » social au sein d'une génération, d'autant qu'elle recoupe les différences de classe sociale et les expériences de racialisation. En ce domaine, les nombreux récits de drague de rue durant les entretiens rendaient tout à fait perceptibles ces frontières sociales entre une jeunesse racisée des quartiers et une jeunesse blanche des centresvilles :

\section{Entretien avec Mounir}

La jeunesse de Strasbourg elle va là-bas. Et avant, moi j'avais les cheveux longs jusqu'à là. J'ai coupé là il y a trois semaines. Jusqu'à là les cheveux. Je les ai coupés. Tac, chemise ouverte, tac tac clac clac, tu vas, tu la bloques. Elles sont sous alcool les 
petites. Juliette, tac tac. Comment tu t'appelles ? T'inquiète, Julien. Alors que je m'appelle Mounir. T'es obligé de leur mentir.

\section{Pourquoi?}

Parce que tu dis je m'appelle Mounir... Moi je veux pas trop me griller. Je passe avec mon pote Benazouz, c'est fini frère. C'est fini, t'es mort dans le film. Tu t'appelles comment toi? Mustapha toi, Billal toi, Youssouf. Ça passe mieux Benoît, Julien, Christian. Tu vois, ça passe cash.

\section{Du coup vous dites ça?}

Mais ouais, obligé. Quand j'avais les cheveux longs, j'avais une petite tête d'Italien tu vois. Je disais: Pedro, Paquito. La vie de ma mère, il y en a une je lui ai dit je m'appelais Juan, Juan da Silva je lui dis, du Sud-Ouest de l'Espagne. Elle m'a dit: «T'habites où? ». Je lui dis : «A Medellin» » Medellin c'est en Colombie » elle me fait. Ah là là, ça ça me fait trop rire !

On notera par ailleurs que le fait d'être incarcéré ne saurait à lui seul expliquer cette démarcation. Typiquement, ceux qui ont été très tôt écartés de l'école auront tendance à se voir comme « en dehors » de la jeunesse, tandis que ceux qui fréquentaient encore l'école et en partagent les codes auront tendance à davantage s'inclure dans la jeunesse à laquelle ils pensent appartenir. Et cet écart se ressent d'autant plus sur la question des relations amoureuses, puisque les jeunes scolarisés auront tendance à fréquenter des filles dans leur groupe d'amis, alors que les autres seront davantage dans une sociabilité marquée par un entre-soi masculin. Pour les jeunes qui étaient scolarisés, les rencontres se faisaient d'ailleurs souvent sur le mode assez classique désormais de la drague via les réseaux sociaux d'une fille connue dans leur établissement. Pour les autres, les rencontres se font essentiellement dans le quartier, ou dans des conditions totalement extérieures, par exemple auprès de travailleuses du sexe (notamment pour les jeunes habitant à proximité de frontières avec des pays ayant libéralisé le travail du sexe). Enfin, on pouvait retrouver chez certains détenus ayant eu une scolarité plus longue (lycée en filière générale, ou en bac pro dans les filières commerciales par exemple) des formes de mépris ayant cours en milieu scolaire pour celles et ceux placés dans les filières de relégation («ici, c'est des SEGPA »).

Le rapport de ces jeunes à l'école est donc resté central, alors même que l'on pourrait penser que celle-ci se situe de facto «loin » d'eux. En fait, ils sont souvent dans une "culture anti-école » (Willis, 1978) exacerbée, mais dans le même temps, c'est l'école qui leur sert encore de principe de vision et division du monde à de multiples occasions. Leurs cursus scolaires souvent interrompus ou chaotiques font écho à leurs parcours judiciaires et s'insèrent de cette façon dans le récit de soi comme déviant ou comme « mauvais » jeune au regard des institutions et plus largement de la société.

\section{Trajectoires affectives et effets de l'incarcération}

31 C'est via notre objet principal d'enquête, soit le rapport à la sexualité, que nous pouvons peut-être le mieux mettre en lumière l'effet de l'incarcération, même courte, sur une trajectoire individuelle, et finalement situer ces jeunes détenus dans l'espace social, notamment par rapport aux autres jeunes de leur génération. Tout d'abord, il apparaît que ces jeunes ont des parcours d'entrée dans la sexualité et dans les relations 
assez typiques de leur milieu social et relativement semblables à ceux des jeunes de manière générale.

Tableau 2. Tableau synthétique des parcours amoureux et sexuels

\begin{tabular}{|c|c|c|}
\hline & Garçons $(n=60)$ & Filles $(n=12)$ \\
\hline $\begin{array}{ll}\text { Situation } & \\
\text { amoureuse au } \\
\text { moment de } \\
\text { l'entretien }\end{array}$ & $\begin{array}{l}\text { Parmi ceux qui déclarent une situation amoureuse } \\
\text { (n=51), près de la moitié des garçons (27) sont } \\
\text { célibataires au moment de l'entretien. } 5 \\
\text { entretiennent des relations ponctuelles, } 7 \text { sont en } \\
\text { couples récent (moins de } 6 \text { mois) et } 12 \text { sont en } \\
\text { couple depuis plus de } 6 \text { mois (dont } 2 \text { qui } \\
\begin{array}{l}\text { entretiennent, par ailleurs, des relations } \\
\text { ponctuelles). }\end{array}\end{array}$ & $\begin{array}{l}3 \text { filles sur } 4 \text { (8) déclarent } \\
\text { être célibataires, le quart } \\
\text { restant déclarant être en } \\
\text { couple depuis plus de } 6 \text { mois. }\end{array}$ \\
\hline $\begin{array}{l}\text { Âge au } \\
\text { premier baiser }\end{array}$ & $\begin{array}{l}\text { L'âge au premier baiser déclaré par les garçons se } \\
\text { situe entre } 2 \text { et } 15 \text { ans, la moyenne étant à 10,5 ans. } \\
\text { Un seul garçon déclare n'avoir jamais embrassé. }\end{array}$ & $\begin{array}{l}\text { L'âge au premier baiser } \\
\text { déclaré par les filles se situe } \\
\text { entre } 10 \text { et } 15 \text { ans, la } \\
\text { moyenne étant à } 12,9 \text { ans. } 3 \\
\text { des } 12 \text { filles déclarent n'avoir } \\
\text { jamais embrassé. }\end{array}$ \\
\hline $\begin{array}{l}\text { Âge au } \\
\text { premier } \\
\text { rapport sexuel }\end{array}$ & $\begin{array}{l}\text { Les garçons sexuellement actifs avaient en } \\
\text { moyenne } 13,5 \text { ans à leur premier rapport sexuel. }\end{array}$ & $\begin{array}{l}\text { Les filles sexuellement } \\
\text { actives avaient en moyenne } \\
14,25 \text { ans à leur premier } \\
\text { rapport sexuel. }\end{array}$ \\
\hline Dépistage VIH & $\begin{array}{l}\text { Parmi les garçons, } 16 \text { déclarent n'avoir jamais eu } \\
\text { recours au dépistage du VIH, } 36 \text { disent y avoir eu } \\
\text { recours (dont } 23 \text { à l'extérieur de la prison et } 13 \mathrm{au} \\
\text { sein de la prison, le plus souvent lors de leur } \\
\text { arrivée). }\end{array}$ & $\begin{array}{l}\text { Parmi les filles, } 10 \text { déclarent } \\
\text { avoir eu recours au dépistage } \\
\text { en prison, } 1 \text { déclare y avoir } \\
\text { eu recours à l'extérieur, et } 1 \\
\text { déclare n'y avoir jamais eu } \\
\text { recours. }\end{array}$ \\
\hline
\end{tabular}

En ce qui concerne la ou plutôt les "premières fois ", le tableau ci-dessus indique que l'entrée dans une sexualité active se fait aux alentours de 13 ans et demi pour les garçons et de 14,25 ans pour les filles (voir pour comparaison Choquet et Hassler, 2003)

Dans une approche qualitative, nous nous sommes surtout intéressés au processus par lequel les jeunes débutaient une vie sexuelle. Nous leur demandions la plupart du temps de raconter cette première fois. Il en ressort, comme en population générale, une certaine confusion des récits, voir un changement de version au cours de l'entretien. Plus spécifiquement, on note chez ces jeunes une prédominance des premières fois accompagnées ou incitées par les pairs, notamment les pairs plus âgés de la «bande de potes » ou des "gars du quartier ». Cette observation rappelle que ces jeunes sont très rarement isolés et marginaux dans leur milieu. Ils ont une surface sociale relativement importante et, fréquemment, sont insérés dans des sociabilités qu'ils jugent valorisantes, avec des jeunes souvent plus vieux qu'eux. 
range dans la catégorie des « putes $^{13}$ ", alors que lui était âgé de 12 ans :

Je traîne avec les grands, et les grands ils m'ont dit: «Wesh, Moussa t'as déjà niqué ? ». J'ai dit : «Beh non... ». Ils m'ont dit : «Vas-y, arrête de mentir ! ». Je dis : «Wallah j'ai pas niqué ! », et wallah ils se foutaient de ma gueule. Après ils m'ont dit : «Beh viens, on va voir truc ». Après on est partis, après je l'ai niquée.

Ces premières fois sous l'impulsion des "grands", donc des amis plus vieux, peuvent d'ailleurs avoir un effet assez rédhibitoire pour des rapports sexuels ultérieurs. Ainsi, Moussa se dit désormais désintéressé par la sexualité, alors qu'il n'a pas voulu avoir d'autres expériences après celle-ci. Pour lui, la sexualité est désormais seulement synonyme de " problèmes » (judiciaires et familiaux) :

Non, parce que moi je trouve que ça sert à rien de niquer wesh, ça sert à rien de niquer. Tu vas niquer pourquoi ? Vas-y, vas-y, tu vas niquer, tu vas faire un gosse.

Tu vas faire un enfant sans faire exprès tu vas gâcher ta vie, ça sert à rien.

Pour d'autres jeunes rencontrés, la première fois doit être réservée à une relation durable et stable, avec souvent le mariage comme condition préalable. Cette règle morale est aussi énoncée dans les cas de jeunes qui ont peu ou pas encore de vie sexuelle active. En particulier chez les garçons, un tel discours peut permettre de contourner la question de la virginité.

Une autre modalité d'entrée dans la sexualité qui est apparue de façon assez récurrente chez ces jeunes est le recours aux prostituées. Dans ce cas, les premières fois ressemblent beaucoup aux initiations forcées (décrites par Moussa plus haut) par les pairs plus âgés avec une fille de leur entourage considérée comme «une pute » et qui aura des relations sexuelles avec plusieurs membres du groupe. Il nous semble important d'insister sur ce mode d'entrée dans la sexualité parce que les travailleuses du sexe sont relativement peu visibles dans les enquêtes sur la sexualité des jeunes, lesquelles se focalisent davantage sur les sites de rencontre comme nouveau phénomène. Il apparaît que ces deux dimensions se répondent, puisque les garçons vont souvent sur des sites où des jeunes femmes (mineures et majeures) postent des annonces de prostitution. Plus largement, dans les établissements pénitenciers situés non loin de pays où la prostitution est plus répandue et légalisée, «aller au bordel » reste un rite initiatique banal. Pour certains, ce rapport à la sexualité tarifée devient routinier, comme c'est le cas de Redouane, incarcéré en EPM. Sa consommation de prostituées s'est d'abord faite dans le cadre festif des fins de soirée, à l'étranger, avec la bande de copains, puis peu à peu de façon individuelle et routinière à l'aide de l'argent amassé au début de sa carrière délinquante. Comme d'autres détenus, Redouane cumule les relations sexuelles avec celles qu'il nomme « les putes» (les « vraies putes » étant selon lui les travailleuses du sexe, les «putes» étant les filles avec qui il a des relations sexuelles «facilement » et sans engager une relation sérieuse). Néanmoins, il a aussi une relation plus longue et a eu un enfant. Par ailleurs, Redouane distingue certains profils de "filles bien ». Lors des entretiens avec les garçons, ces «filles bien " servent d'abord à justifier les catégories stigmatisantes des "putes", qui sont donc parfois catégorisées comme telles seulement parce qu'elles ont eu des relations sexuelles «avec eux». Puisqu'eux-mêmes se considèrent souvent comme des «cas sociaux » ou des "délinquants ", une fille qui s'intéresse à eux sera de fait stigmatisée elle aussi. Les «filles bien» (celles qui précisément ne sortent pas avec eux) sont davantage inatteignables et les principes religieux de virginité au mariage jouent aussi en ce sens.

Populations vulnérables, 4 | 2018 
38 Mais les «filles bien» sont aussi celles avec qui ces jeunes disent vouloir se marier, faire des enfants, fonder une famille stable et conforme. Cette volonté d'inscrire le ou la partenaire du côté de la jeunesse respectable était très présente en effet, du moins chez les jeunes qui se disaient "en couple». Nous parlons ici d'environ un quart des enquêtés (filles et garçons) dans une relation durable entamée avant la détention. Il faut noter ici que l'effet de l'incarcération sur les trajectoires amoureuses et conjugales est très important. Il accentue les suspicions au sein des couples, aussi certains détenus mettent-ils en place des dispositifs de surveillance de leur petite amie, via les amis ou la famille. La jalousie semble ainsi centrale dans leurs communications par téléphone ou au parloir lorsque la compagne dispose d'une autorisation de visite. Par exemple, un détenu en EPM, vivant en couple dans un logement avec sa compagne depuis un an, demande à cette dernière de "se laisser pousser les poils aux jambes» et de les lui montrer au parloir en gage de sa fidélité. Dès lors, la question de savoir si la partenaire est une « fille bien » se pose avec d'autant plus d'importance durant l'incarcération. En lien, les entretiens auront révélé comment l'incarcération les conduit à recomposer leurs attentes vis-à-vis des relations amoureuses, en «laissant tomber » une relation, ou au contraire en « s'attachant » à une amie qui leur écrit régulièrement.

\section{Conclusion}

En guise de conclusion et d'ouverture, il nous semble intéressant de penser les effets de l'incarcération sur les trajectoires en fonction des modalités selon lesquelles ces jeunes se projettent dans l'avenir. Malgré la diversification croissante des formes de constitution du couple et de la famille (Stroebel, 2008 ; Renaud et Ogg, 2009), les jeunes rencontrés continuent d'aspirer à une vision du « modèle familial idéal » dans lequel les enfants naissent dans un couple stable, uni par les liens du mariage. La conformité est ainsi essentielle aux désirs de réussite sociale, et montre qu'ils séparent assez clairement leur condition présente de celle de leur vie "plus tard », quand ils auront selon eux, très unanimement, des enfants, une compagne et une maison. Cette évocation constante d'une volonté de s'installer dans un modèle familial conforme et donc respectable (Bourgois, 2002) sert aussi à justifier le fait qu'ils n'ont pour le moment pas les moyens d'y accéder, et donc qu'ils n'ont pas à se comporter de manière à s'installer durablement sur le marché matrimonial. Nous sommes donc là sur un domaine de la vie où ces jeunes s'inscrivent pleinement dans les caractéristiques de leur génération en population générale. Dans le même temps, leurs façons d'envisager une éventuelle stabilité montrent la spécificité des mineurs détenus par rapport aux manières de «devenir adulte " propres à leur génération et au contexte social dans lequel ils évoluent. Leurs trajectoires sont en effet caractérisées par un brouillage des étapes (Van de Velde, 2015) bien plus fort que pour les autres du même âge (RégnierLoillier, 2009 ; 2016): ces jeunes qui souvent "gagnent leur vie» (s'assument financièrement en tout cas) bien avant les autres adolescents insistent aussi sur le fait de vouloir vivre leur jeunesse hors d'un carcan conjugal et expriment dans l'ensemble leur peur d'avoir des enfants. Sur ce point, leur situation présente leur permet de justifier qu'ils ne sont pas en mesure d'assumer un enfant, mais qu'ils le seront à l'avenir (ce qui d'ailleurs les rend globalement favorables à l'avortement). Quant à ceux qui sont déjà parents, ils pointent les inconvénients de cette situation et les difficultés inhérentes à leur relation avec la mère alors qu'ils sont incarcérés. Plus largement, l'insistance de ces jeunes à se projeter dans l'avenir via la conjugalité rappelle quels 
domaines de la vie leur semblent peu propices à une éventuelle "réussite " sociale. Le travail apparaît comme secondaire. L'école n'est même plus présente pour une partie d'entre eux. Dès lors, il leur reste des ressources incorporées permettant de séduire et de se projeter dans une vie conjugale, marquée par une forte solidarité familiale. Leurs récits montrent alors, en général, que l'incarcération participe d'un processus de stigmatisation et d'auto-exclusion. Ces jeunes ont tendance à se percevoir eux-mêmes comme des jeunes à part, ou en dehors de la jeunesse. Celle-ci est caractérisée selon eux, avant tout, par une scolarisation longue et des pratiques festives légitimes, mais aussi par une condition sociale plutôt située du côté des classes moyennes et non racisées ${ }^{14}$. Autrement dit, et pour reprendre les mots d'un jeune interrogé durant l'enquête, «les jeunes", ce sont «les étudiants [y compris lycéens], tranquilles, du centre-ville, qui ont des sacs Eastpack ».

\section{BIBLIOGRAPHIE}

Amsellem-Mainguy Y. et Dumollard D. (2015), « Santé et sexualité des jeunes pris en charge par la PJJ : entre priorité et évitement », Rapport de recherche, INJEP.

Bajos N. et Bozon M. (2008), Enquête sur la sexualité en France. Pratiques, genre et santé, Paris, La Découverte.

Beaud S. et Pialoux M. (2003), Violences urbaines, violences sociales. Genèse des nouvelles classes dangereuses, Paris, Fayard.

Berger D., Rochigneux J.-C., Bernard S., Morand J. et Mougniotte A. (2015), « Éducation à la sexualité : conceptions des élèves de $4^{\mathrm{e}}$ et de $3^{\mathrm{e}}$ en collège et SEGPA », Santé Publique, vol. 27, $\mathrm{n}^{\circ} 1$, p. $17-26$.

Bessin M., Bidart C. et Grossetti M. (dir.) (2010), Bifurcations. Les sciences sociales face aux ruptures et à l'évènement, Paris, La Découverte.

Bonelli L. (2003), « Évolutions et régulations des illégalismes populaires en France depuis le début des années 1980 », Cultures \& Conflits, n 51, p. 9-42.

Bony L. (2015), « La prison, une "cité avec des barreaux" ? Continuum socio-spatial par-delà les murs », Annales de géographie, n 702-703, p. 275-299.

Bourgois P. (2002), In Search of Respect. Selling Crack in El Barrio, New York, Cambridge University Press.

Chantraine G. (2008), Trajectoires d'enfermements. Récits de vie au quartier mineur, Saint-Quentinen-Yvelines, CESDIP.

Clair I. (2008), Les jeunes et l'amour dans les cités, Paris, Armand Colin.

Douat É. (2011), L'école buissonnière, Paris, La Dispute.

Fréchon I. et Robette N. (2013), « Les trajectoires de prise en charge par l'ASE de jeunes ayant vécu un placement ", Revue française des affaires sociales, $n^{\circ}$ 1-2, p. 123-145. 
Goffman E., 1968, Asiles. Études sur la condition sociale des malades mentaux, Paris, Minuit.

Goyette M. et Fréchon I. (2013), « Comprendre le devenir des jeunes placés : la nécessité d'une observation longitudinale et représentative tenant compte des contextes socioculturel et politique ", Revue française des affaires sociales, $n^{\circ} 1-2$, p. 165-182.

Jobard F. et Levy R. (2010), «Les contrôles d'identité à Paris ", Questions pénales, vol. XXIII, n 1.

Le Caisne L. (2008), Avoir 16 ans à Fleury. Une ethnographie d'un centre de jeunes détenus, Paris, Seuil.

Lepoutre D. (1997), Cour de banlieue. Codes, rites et langages, Paris, Odile Jacob.

Maillochon F. (2001), « L'âge des amours. Différence d'âge entre partenaires et construction du genre au moment de l'initiation sexuelle », Europcea, n 1-2, p. 47-64.

Mauger G. (2006), Les bandes, le milieu et la bohème populaire. Études de sociologie de la déviance des jeunes des classes populaires, Paris, Belin.

Millet M. et Thin D. (2005), Ruptures scolaires. L'école à l'épreuve de la question sociale, Paris, PUF.

Palheta, U. (2012), La domination scolaire. Sociologie de l'enseignement professionnel et de son public, Paris, PUF.

Régnier-Loilier Arnaud (dir.) (2009), Portraits de familles. L'enquête Étude des relations familiales et intergénérationnelles, Paris, INED.

Régnier-Loiler A. (dir.) (2016), Parcours de familles. L'enquête Étude des relations familiales et intergénérationnelles, Paris, INED.

Sallée N. (2016), Éduquer sous contrainte. Une sociologie de la justice des mineurs, Paris, EHESS.

Teillet G. (2016), «L'intervention judiciaire auprès des mineurs », revue de littérature INJEP. En ligne : http://injep.fr/publication/lintervention-judiciaire-aupres-des-mineurs/.

Van de Velde C. (2015), Sociologie des âges de la vie, Paris, Armand Colin.

Vuattoux A. (2014), «Adolescents, adolescentes face à la justice pénale », Genèse, vol. 4, n 97, p. 47-66.

Wacquant L. (2004), Punir les pauvres, le nouveau gouvernement de l'insécurité sociale, Marseille, Agone.

Willis P. (1978), "L'école des ouvriers ", Actes de la recherche en sciences sociales, vol. 24, novembre 1978, p. 50-61.

\section{NOTES}

1. Cette enquête a reçu le soutien de la Direction générale de la santé, de la Direction de la protection judiciaire de la jeunesse, de la Direction de l'administration pénitentiaire, et a été réalisée par l'INJEP.

2. Les entretiens ont toujours été réalisés avec l'un ou l'une des sociologues, dans des lieux préservant la confidentialité (salles fermées, sans présence extérieure) et sur le principe du volontariat. Ils étaient enregistrés et ont ainsi pu faire l'objet d'une analyse approfondie. Les enquêtés (jeunes et professionnels) avaient la possibilité d'interrompre l'entretien à tout moment ou de ne pas répondre à certaines de nos questions, ce qui ne s'est que très rarement produit. Les entretiens ont duré entre 45 minutes et 3 heures.

3. Les prisons, tout comme les prénoms des enquêtés, sont rendus anonymes dans le présent chapitre, afin de garantir la confidentialité des propos tenus lors de l'enquête. 
4. Pour en savoir plus sur le fonctionnement des EPM, voir la thèse de Laurent Solini et le rapport dirigé par Gilles Chantraine, intitulé « Les prisons pour mineurs. Controverses sociales, pratiques professionnelles, expériences de réclusion », Lille, Clersé, GIP Justice, 2011.

5. Données fournies et calculées par le bureau des études (SDME-Me5).

6. Ces données sont extraites des statistiques mensuelles des personnes écrouées et détenues, Direction de l'administration pénitentiaire, Bureau des études (SDME-Me5), situation au $1^{\text {er }}$ mai 2017.

7. Ministère de la justice, Les condamnations 2011, 2013 [Données du Casier judiciaire national].

8. Nous ne disposons pas de statistiques sur la race, donc nous nous tenons à des observations relatives et aux entretiens menés avec les jeunes et les professionnels abordant cette question. $A$ minima pouvons-nous nous appuyer sur les données relevées par Fabien Jobard et René Levy à propos des contrôles d'identité (Jobard et Levy, 2010), c'est-à-dire sur l'amont de la chaîne pénale, ainsi que sur le travail mené par Léonore Le Caisne à Fleury Mérogis en 2008.

9. Selon les établissements où nous avons enquêté, la proportion de jeunes racisés était variable, de très majoritaire dans le $\mathrm{QM}$ de région parisienne, à relativement majoritaire, voir minoritaire dans les EPM et QM que nous avons visité ailleurs en France. Par contre, dans tous les établissements, les détenus appartenaient à différentes fractions des classes populaires, jamais aux classes supérieures ou même intermédiaires (les professionnels enquêtés soulignent d'ailleurs le caractère rare et exceptionnel de l'incarcération de jeunes d'autres milieux sociaux que les classes populaires).

10. Nous sommes là sur des situations bien connues des prisons pour majeurs, et qui par ailleurs concernent surtout les garçons étant donné le peu d'établissements de détention pour jeunes filles mineures.

11. «Auxi » correspond à la fonction «d'auxiliaire ", c'est-à-dire à une responsabilité « accordée » par l'Administration pénitentiaire à certains détenus, correspondant à des tâches de nettoyage ou de logistique au sein d'une unité.

12. Ce type d'émission de téléréalité met en scène des jeunes qui partagent une villa dans un pays étranger.

13. Selon les catégories de division du monde entre la «pute » et la "fille bien » avec qui l'on a parfois peu ou pas de rapports sexuels, et avec laquelle les garçons se projettent parfois dans l'avenir.

14. Par "racisées", nous entendons ici les populations construites comme telles par un processus social d'assignation de certaines populations à une origine nationale, ethnique ou raciale réelle ou perçue, à une couleur de peau et/ou une religion. Les " non racisés " sont alors, dans le discours des jeunes, les «blancs », parfois également appelés les « Français ».

\section{RÉSUMÉS}

Quel lien peut-on faire entre les trajectoires des mineurs incarcérés et leurs plus ou moins grandes vulnérabilités face à l'expérience de l'incarcération? À partir d'une enquête par entretiens auprès des mineurs incarcérés et des professionnels qui les encadrent, l'article étudie les conséquences des ruptures biographiques, scolaires et amoureuses induites par la ou les incarcérations successives. Les jeunes rencontrés affichent sur ces différents aspects des différences notables, liées notamment aux parcours scolaires inégaux. Les « décrocheurs » sont les plus prompts à se situer comme «en dehors» de la jeunesse. Tandis que ceux qui 
fréquentaient encore l'école au moment de leur incarcération partagent davantage les codes de leur génération et voient plus la prison comme un stade transitoire, qui ne marquera que leur jeunesse, mais pas forcément leur «vie d'adulte ». L'autre thématique abordée dans l'article est celle de l'entrée dans la sexualité. Sur ce point, les jeunes filles et garçons rencontrés sont assez semblables à celles et ceux de leur milieu social. En revanche, l'incarcération est une épreuve dans la gestion de leurs relations amoureuses, amicales et familiales. L'emprisonnement tend à pousser ces jeunes à recomposer leurs attentes en termes de relations amoureuses mais aussi amicales. Plus généralement, cette expérience favorise un rapport aux autres marqués par la suspicion et l'auto-exclusion.

This article studies the consequences of incarceration on the social and academic trajectories as well as romantic lives of incarcerated youth. What might be the link between their social trajectories and experiencing more or less vulnerability during incarceration? The research presented relies on a series of interviews conducted with incarcerated youth and the professional staff in charge of them. We see notable differences among the youth linked particularly to divergent educational backgrounds. The "décrocheurs" (the "drop-outs"), are those most likely to see themselves as being "external" to youth. While those who are still in school when incarcerated share the codes and norms of their generation and see prison only as a transitory stage that will mark their youth but not their adult life. The second topic discussed in this article concerns incarcerated youth's experiences with becoming sexually active. On this matter, incarcerated girls and boys share a similar experience with the non-incarcerated youth with whom they share a similar social background. But incarceration is an ordeal that forces them to reevaluate their expectations when it comes to romantic relationships and friendships. This experience is characterized by forms of suspicion towards others and self-exclusion.

\section{INDEX}

Mots-clés : jeunesse, santé, sexualité, prison

Keywords : youth, health, sexuality, prison

\section{AUTEURS}

\section{YAËLLE AMSELLEM-MAINGUY}

Chargée de recherche

Institut national de la jeunesse et de l'éducation populaire - INJEP

amsellem-mainguy@injep.fr

\section{BENOÎT COQUARD}

Institut national de la recherche agronomique - INRA

\section{ARTHUR VUATTOUX}

École des hautes études en santé publique - EHESP, Institut national de la jeunesse et de l'éducation populaire - INJEP

vuattoux@univ-paris13.fr 